\title{
The Adequacy of the Present Practice in Dynamic Aggregated Modeling of Wind Farm Systems
}

\author{
Linash Puthenpurayil Kunjumuhammed, Senior Member, IEEE, Bikash Chandra Pal, Fellow, IEEE, \\ Colin Oates, Member, IEEE, and Kevin J. Dyke, Member, IEEE
}

\begin{abstract}
Large offshore wind farms are usually composed of several hundred individual wind turbines, each turbine having its own complex set of dynamics. The analysis of the dynamic interaction between wind turbine generators (WTG), interconnecting ac cables, and voltage-source converter (VSC)-based high voltage DC (HVDC) system is difficult because of the complexity and the scale of the entire system. The detailed modeling and modal analysis of a representative wind farm system reveal the presence of several critical resonant modes within the system. Several of these modes have frequencies close to harmonics of the power system frequency with poor damping. From a computational perspective, the aggregation of the physical model is necessary in order to reduce the degree of complexity to a practical level. This paper focuses on the present practices of the aggregation of the WTGs and the collection system, and their influence on the damping and frequency characteristics of the critical oscillatory modes. The effect of aggregation on the critical modes is discussed using modal analysis and dynamic simulation. The adequacy of aggregation method is discussed.
\end{abstract}

Index Terms-Aggregation, DFIG, high voltage DC (HVDC), stability, voltage-source converter (VSC), wind farm.

\section{INTRODUCTION}

$\mathbf{L}$ ARGE offshore wind farms are increasingly being installed to commercially harness wind energy.The operation of such large wind farms involve high voltage DC (HVDC) transmission to transport the power to shore; however this is reportedly facing a major technical challenge with the AC waveform in the offshore wind farm becoming too distorted for the wind turbines to lock. The waveform distortion problem may occur due to technical problems within the wind farm, the power collector system, the voltage-source converter (VSC) based HVDC link or an inherent instability within the grid.

In [1], the stability analysis of a wind farm system having 136 wind turbine generators (WTGs) is presented using a detailed representation of the generator and collector system. The result shows the presence of three medium frequency modes (MFM) and stator modes in the wind farm system with poor damping.

Manuscript received October 6, 2015; revised February 11, 2016, April 17, 2016, and April 28, 2016; accepted April 28, 2016. Date of publication June 7, 2016; date of current version December 14, 2016. This work was supported by EPSRC, U.K. under Grant Stability and Control of Power Networks with Energy Storage UK-China Grid Scale Storage EP/L014343/1. Paper no. TSTE00831-2015.R2.

L. P. Kunjumuhammed and B. C. Pal are with the Department of Electrical and Electronic Engineering, Imperial College, London SW7 2AZ, U.K. (e-mail: linash.p.k@imperial.ac.uk; b.pal@imperial.ac.uk).

C. Oates, deceased, was with the GE's Grid Solutions, Stafford ST17 4LX, U.K. (e-mail: colin.oates@alstom.com).

K. J. Dyke is with the GE's Grid Solutions, Stafford ST17 4LX U.K. (e-mail: kevin.dyke@ge.com).

Digital Object Identifier 10.1109/TSTE.2016.2563162
The MFMs were shown to have frequencies close to harmonics of the power system frequency and the stator modes having frequencies close to power frequency. It is realized that the frequency and damping of these modes are influenced by the operating conditions and the VSC controller tuning.

The results are obtained using detailed modelling of the WTGs and the collector system. The non-linear dynamic model developed contains 3436 ordinary differential equations and its linearised model had 1273 pairs of complex eigenvalues. The analysis of such detailed and complex model by conventional method for transient and small signal stability study is impractical.

A more appropriate approach for dynamic simulation of wind farm is to use aggregated model of WTGs and the collector system [2]. However, a study using an aggregated wind farm system (AWS) is likely to influence the system properties due to the possibility of the aggregation process masking many of the internal resonant modes within the wind farm or altering their characteristics. The objective of this paper is to the assess impact of the aggregation on the characteristics of internal wind farm oscillatory modes.

Various methods for wind farm aggregation are presented in Section II and the selection of suitable approach to address the current problem is discussed. An AWS is developed from the full wind farm system (FWS) described in [1]. A simulation model is developed for the AWS and the analysis of critical modes is presented in Section III through eigenvalue analysis and participation factor $(\mathrm{PF})$. Comparison between the characteristics of critical modes present in the FWS and the AWS are discussed, and the dynamic simulation results are presented in Section IV.

\section{AgGregation OF Wind FARM}

Modern wind farms contain hundreds of WTGs and several strings of cables forming the collector system network. Generally the layout of collector system network within wind farms are not identical [3]-[5]. Also, the operating condition of individual WTGs in a wind farm is affected by the prevailing wind speed, direction, wind farm layout and grid operating conditions [6]-[8].

In many studies, the wind farm aggregation is focused on obtaining the behaviour of the wind farm at the point of common coupling (PCC) during the normal operation and grid disturbances alone. A fully aggregated model of wind farm is proposed by many authors which are claimed to be adequate to represent general dynamic behaviour of a wind farm at PCC [10]-[12]. In a fully aggregated model the wind farm is represented by 
one equivalent wind turbine and generator. The capacity of the aggregated machine is the sum of individual WTG capacities. In [13], dynamic simulations are carried out to establish relationship between wind farm operating conditions and power system, and aggregation is carried out under worst operating condition of the wind farm.

However, a fully aggregated model is not adequate when the wind speed across a wind farm varies and many WTGs operate below their rated condition. A semi aggregated model is proposed in [14]-[18] to account for changes in wind speed where the individual wind turbines are modelled and their aggregate output is fed to an aggregated generator. In [19], authors argue that the use of average rotor speed for the aggregated generator can cause some discrepancies and the performance of the semi aggregated model can be improved by using a mechanical torque compensation factor.

One aggregated generator and an aggregated collector system impedance, however, cannot capture diversity in the collector system voltage, dynamics of different types of WTGs used in a wind farm, or variation in output of WTGs due to wake effect in large wind farms [7]. A multi machine modelling approach where more than one aggregated machine is used to represent a wind farm is more appropriate solution. WTGs having the same incoming wind speed are aggregated in [11], [20]. Further improvement is proposed [21] in which wind power curve is divided into many sections and the WTGs fall in one section are aggregated. In [22], post fault voltage profile in the collector network is used to determine number of aggregate machines. In [23], [24], the authors propose a coherency based approach to find WTGs with similar behaviour and such coherent groups are replaced with an equivalent unit. WTGs of specific technology are grouped separately in [25] to form an aggregated wind farm model.

Output of the aggregated turbine and generator are calculated by equivalent wind method in many literatures [11], [14], [26], [27]. In this method, the output power of each wind turbine is obtained from the incident wind and the output power curve, and sum of the outputs is equal to aggregated WTG output. An equivalent output power curve is used to calculate the equivalent wind incident on the aggregated WTG. The aggregated wind turbine model is identical to individual WTG model employing the same electrical and mechanical parameters in per unit value under the respective machine bases [28]. In [29] author states that the parameters of equivalent model must be adaptive to stochastic changes in the wind farm operating conditions. A recursive identification is proposed to continuously tune the parameters of the dynamic equivalent model of the aggregated machine. Methods based on probabilistic clusturing [26], balanced truncation [30] and heuristic approximation [31], [32] are also proposed.

Another important aspect of wind farm aggregation is the accurate and adequate representation of the collector system network. For a fully aggregated model the entire collector system is represented using an impedance, and number of aggregated impedances depend on the number of aggregated generator. The equivalent impedance is calculated by comparing short circuit impedance [17], [18] or power losses [2], [20] between the full wind farm network and the aggregated wind farm network.

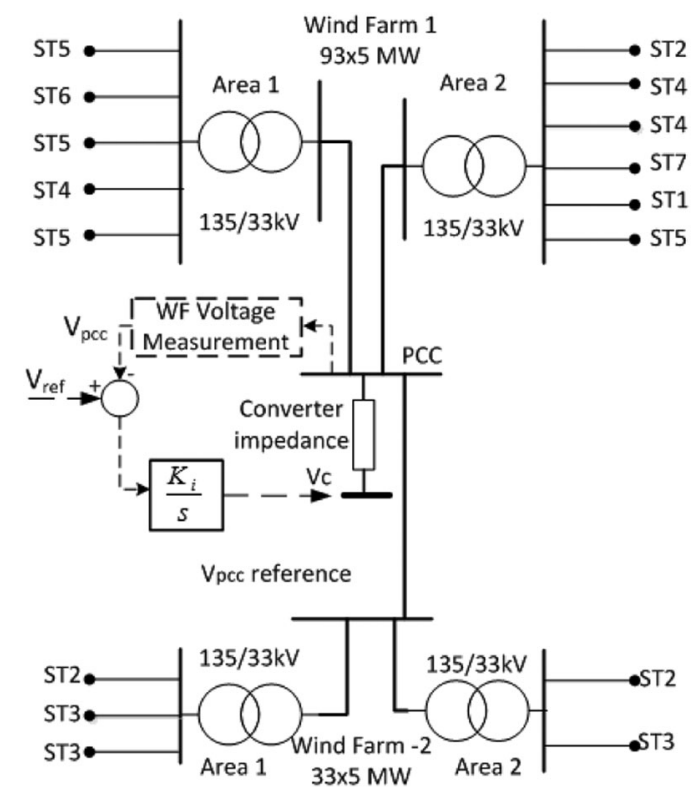

Fig. 1. Single line diagram showing high voltage side of the FWS.

The collector cable impedance is neglected as it is small when compared to the transformer impedance [14]. In [22] authors compare post-short circuit voltage profile in the full wind farm network to adequately represent voltage profile diversity in the collector system.

\section{A. Selection of Aggregation Method}

The objective of this study is to analyze effect of aggregation on the MFMs observed in a wind farm system using full scale modelling [1]. The modes have participation from states of models of collector system components. The aggregation method should (a) preserve the characteristics of the MFM and (b) reducing the size of the model for ease of simulation and analysis.

A fully aggregated model is not suitable for studying oscillations inside the collector system as it completely eliminates these modes from the model. An alternative is to use multi machine modelling approach where a group of WTGs with some similarity could be aggregated. Aggregating machines having similar wind speed is one of the options proposed. However, in large wind farms such as one discussed in [1], WTGs in close proximity and similar wind speed may be located in different strings of the collector system, and their electrical distance may be large. As a compromise, WTGs located in a string are aggregated. The turbine of the WTGs are also aggregated as the mechanical states do not participate in the collector system oscillatory modes [1], and the wind speed is assumed to be constant for the duration of the simulation. The aggregate impedance of a string is calculated by comparing the apparent power losses.

\section{B. Wind Farm System}

The FWS [1] shown in Fig. 1 contains two wind farms of capacity $465 \mathrm{MW}(93 \times 5 \mathrm{MW})$ and $165 \mathrm{MW}(33 \times 5 \mathrm{MW})$. They are named as Wind Farm 1 (WF1) and Wind Farm 2 


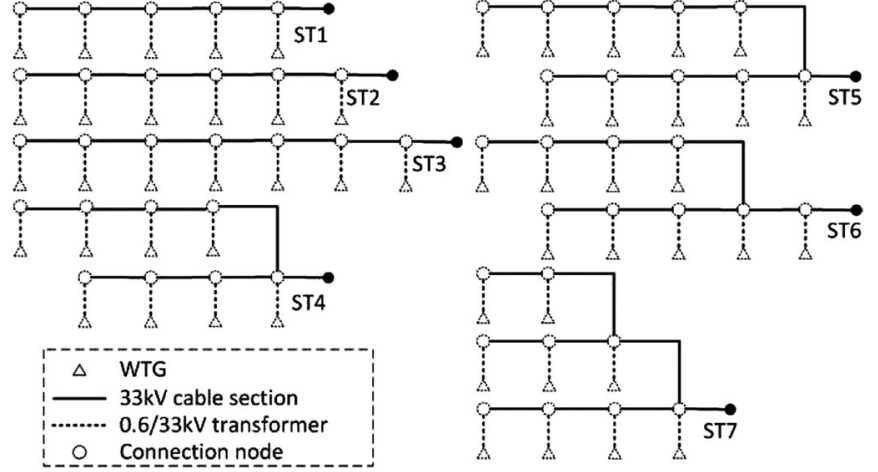

Fig. 2. Structure of the strings in the FWS.

(WF2), respectively. Both the wind farms are divided into two areas, Area-1 (A1), and Area-2 (A2). An HVDC link is used to connect the wind farms to the grid. The wind farm transformers (WFT) and the VSC of the HVDC are connected using $132 \mathrm{kV}$ cables of length $30 \mathrm{~km}$. The interconnection of $132 \mathrm{kV}$ cables and the VSC is called PCC. The VSC transfers energy from the wind farm network to DC line, and controls voltage and frequency at the PCC [33]. The only feedback loop required for the VSC is the voltage magnitude as the frequency and phase angle are predefined. The PCC voltage is compared with a reference voltage and fed to an integral controller as shown in the Fig. 1. The controller gain is tuned such that the transfer function between the reference voltage and the PCC voltage has a gain cross over frequency of $100 \mathrm{~Hz}$.

WTGs are connected to the $33 / 132 \mathrm{kV}$ WFTs using strings of $33 \mathrm{kV}$ cables. Fig. 2 shows configurations of strings (ST1 to ST7) used in FWS. A string contains between 5 and 10 WTGs, and the $33 \mathrm{kV}$ cable length between two WTGs is $1 \mathrm{~km}$. The dotted lines represent $0.6 / 33 \mathrm{kV}$ WTG transformer (WTGtr) and triangles represent the WTGs. Each WTG unit in the FWS is formed of a wind turbine, a DFIG, and a pad mounted transformer. $33 \mathrm{kV}$ cables of two ratings are used in the WF1 and WF2 [1]. In WF1, a $33 \mathrm{kV}$ cable connecting a string to the WFT has a higher capacity compared to the other $33 \mathrm{kV}$ cables. Similarly the WF2 also contains two types of $33 \mathrm{kV}$ cables with lower capacity cables being used to carry power from, at most, three WTGs.

\section{Aggregation of WTGs and Pad-Mounted Transformers}

An AWS is developed by representing each string of the wind farm using an aggregated WTG. Capacity of the aggregated machine is equal to $n X$ capacity of one WTG, where $n$ is the number of WTGs aggregated. Active and reactive power output of individual turbines in a string is aggregated to obtained the output of the aggregated generator and the equivalent wind speed is obtained from an equivalent power curve. The electrical and mechanical parameters of the aggregated machine in per unit are the same as the WTG in respective machine base [2], [28].

The WTGtr model is scaled such that the power losses and voltage drop across the transformer are equal [2]. If $R, L$, and $C$, respectively represent the series equivalent resistance, inductance and capacitance of a pad mounted transformer, and $n$

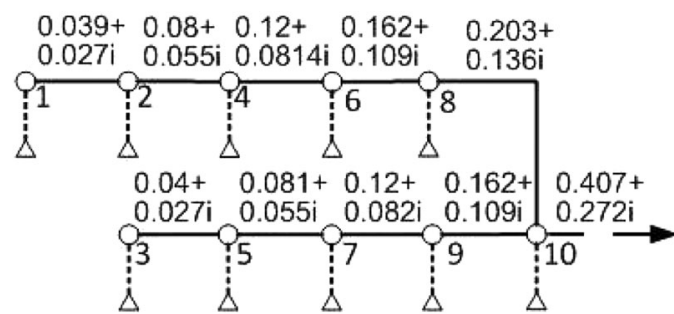

Fig. 3. A WF1 A1 string showing the current flow through the $33 \mathrm{kV}$ cable section.

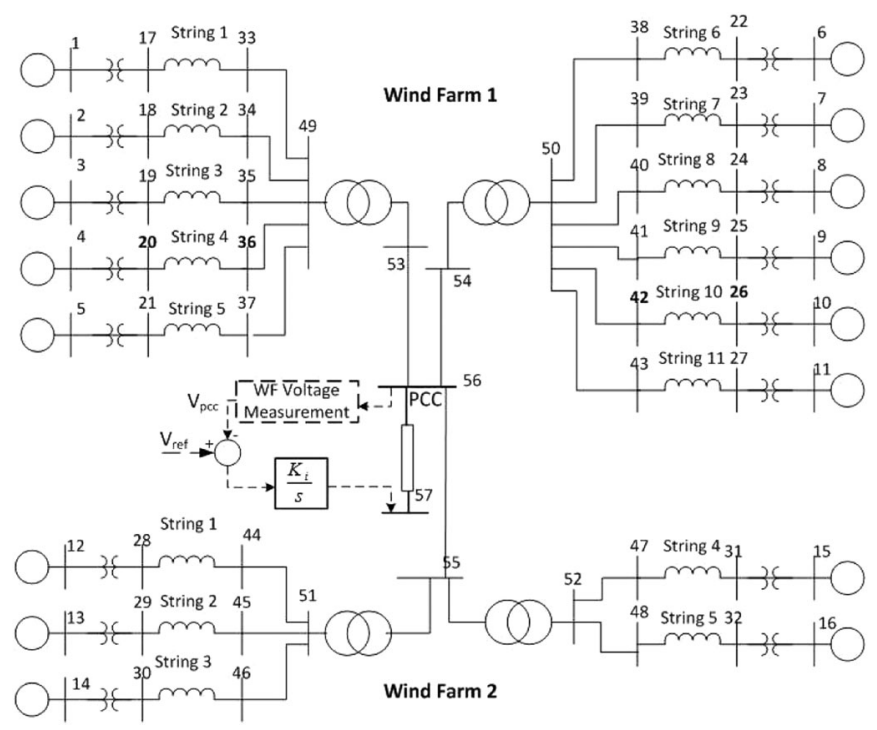

Fig. 4. Aggregated model of the wind farm.

WTGs are aggregated to form a machine, a transformer in the AWS is represented using the parameters $R / n, L / n$ and $C n$.

\section{Aggregation of the Collector Network}

The aggregate impedance of a collector string is obtained by comparing apparent losses in the collector string of the FWS and the AWS. For an example collector string as shown in Fig. 3, let $I_{1}, I_{2}, \ldots I_{10}$ be current injected by the WTGs at buses $1,2 \ldots 10$, respectively. Let us also assume that $I_{1}=I_{2}=\cdots=I_{10}=I$, and impedance of a section is $Z$. The apparent power losses in the string, $S_{T}=I^{2}\left(\sum_{m=1}^{5} m^{2} Z+\sum_{m=1}^{4} m^{2} Z\right)$. Note that the 10th WTG output is feeding to the non-aggregated cable. The current output of the aggregated machine is $I_{a}=10 I$ and apparent power loss through an aggregate impedance $Z_{a}$ would be $I_{a}^{2} Z_{a}$. Hence, $Z_{a}=\left(\sum_{m=1}^{5} m^{2} Z+\sum_{m=1}^{4} m^{2} Z\right) / 10^{2}$. However, current output from all WTGs may not be equal all the time. Hence, instead of using the simplified equation proposed, the apparent power loss in each collector cable section is calculated separately. An illustration of the calculation is given below.

1) Illustration: Fig. 3 shows current flowing through the $33 \mathrm{kV}$ collector cable sections of the WF1 A1. The series impedance of each section is $0.0138+\mathrm{i}^{*} 0.0096 \mathrm{pu}$. The apparent power loss in the string up to bus 10 is $\left(S_{\text {loss }}\right)=0.0007-$ $i^{*} 0.0033 \mathrm{pu}$. The current flowing out of the string from bus 10 is $\left(I_{\text {out }}\right)=0.407+0.272 \mathrm{i}$ pu. The impedance of the equivalent 
TABLE I

CAPACITY OF DFIGS IN THE AWS

\begin{tabular}{lcc}
\hline \hline \multirow{2}{*}{ Location } & Strings & AWS-DFIG capacity \\
\hline WF1 A1 & String-1, String-3, String-5 & $60 \mathrm{MW}$ \\
& String-2 & $45 \mathrm{MW}$ \\
& String-4 & $40 \mathrm{MW}$ \\
WF1 A2 & String-6 & $30 \mathrm{MW}$ \\
& String-7, String-8 & $40 \mathrm{MW}$ \\
& String-9 & $45 \mathrm{MW}$ \\
& String-10 & $25 \mathrm{MW}$ \\
WF2 A1 & String-11 & $50 \mathrm{MW}$ \\
& String-1 & $30 \mathrm{MW}$ \\
WF2 A2 & String-2, String-3 & $35 \mathrm{MW}$ \\
& String-4 & $30 \mathrm{MW}$ \\
& String-5 & $35 \mathrm{MW}$ \\
\hline \hline
\end{tabular}

string $=S_{\text {loss }} / I_{\text {out }}^{2}=0.0117+\mathrm{i}^{*} 0.0081 \mathrm{pu}$. The shunt admittance of each collector cable section $=0.0006 \mathrm{pu}$. The shunt admittance of the equivalent cable $=9^{*} 0.0006=0.0054 \mathrm{pu}$ (nine $33 \mathrm{kV}$ cable sections aggregated).

A schematic representation of the AWS model which contains 16 WTGs is shown in Fig. 4. Each collector string in the original system is represented by an aggregated machine, a WTGtr, an aggregated $33 \mathrm{kV}$ cable, a non-aggregated $33 \mathrm{kV}$ cable. The capacities of the aggregated machines are listed in Table I. No changes are made to the remaining parts of FWS: WTF, $132 \mathrm{kV}$ cables, and VSC. The parameters of the network are given in [1].

\section{E. Wind Farm Modelling}

The wind farm simulation program is organized by merging the models of the WTG, transformer, cable, VSC, and VSC control as shown in Fig. 5. The WTG block contains differential and algebraic equations representing DFIGs and wind turbines. The states inside the WTG block are d-q axis stator currents $\left(i_{\mathrm{ds}}\right.$ and $\left.i_{\mathrm{qs}}\right), \mathrm{d}-\mathrm{q}$ axis stator voltages $\left(e_{\mathrm{ds}}\right.$ and $\left.e_{\mathrm{qs}}\right)$, rotor side converter cascaded PI controller states, grid side converter cascaded PI controller states, and wind turbine mechanical states. A detailed derivation of the DFIG model is reported in many literatures [10]. The transformers (WTGtr and WFT), cables (S1, $\mathrm{S} 2,132 \mathrm{kV}$ ), and VSC are modelled using ' $\Gamma$ ' sections where vertical line indicates sending end capacitance and horizontal line indicates series resistance and inductance. Accordingly, the block representing transformer, cable or VSC has four states such as D-Q axis sending end (across capacitor) voltages $\left(v_{\mathrm{SD}}\right.$ and $v_{\mathrm{SQ}}$ ), and $\mathrm{D}-\mathrm{Q}$ axis receiving end (through inductor) currents $\left(i_{\mathrm{rD}}\right.$ and $\left.i_{\mathrm{rQ}}\right)$. The VSC PI controller (VSCcontrol) has one state each in D and Q axis.

The number above each block in the Fig. 5 indicates number of elements that particular block represents. For example, number 16 above the WTG block means that it represents 16 WTG machines of the AWS. Each state inside the block has size $16 \times 1$, and they are indexed in the order shown in the Fig. 4.

The simulation model of AWS is developed in MATLAB/Simulink software. A linearized model of AWS is obtained using command linmod [34] which returns state matrix $\mathbf{A}$, input matrix $\mathbf{B}$, output matrix $\mathbf{C}$, and feedthrough ma-
TABLE II

THE MFMS IN THE AWS

\begin{tabular}{lccl}
\hline \hline Mode & $\mathrm{f}(\mathrm{Hz})$ & $\zeta(\%)$ & \multicolumn{1}{c}{ Location of participating states } \\
\hline MF1 & 369 & 0.53 & VSC, PCC and WFT \\
MF2 & 257 & -3.2 & VSC, PCC and WFT \\
MF3 & 117 & -19.8 & VSC, PCC and WFT \\
MF4 & 269 & 9.6 & WTGtrs in WF2 and WFTs in WF2 \\
MF5 & 229 & 8.9 & WTGtrs in WF2 and WFTs in WF2 \\
MF6 & 210 & 7.1 & WTGtrs, WFTs and 132 kV cables in WF1 \\
MF7 & 383 & 10 & WTGtrs in WF2 A2 \\
MF8 & 384 & 9.9 & WTGtrs in WF2 A1 \\
MF9 & 391 & 6.6 & 10th WTGtr in WF1 A2 \\
MF10 & 382 & 4.8 & 2nd WTGtr in WF1 A1 \\
MF11 & 388 & 5.8 & 6th WTGtr in WF1 A2 \\
MF12 & 386 & 4.6 & 9th and 11th WTGtrs in WF1 A2 \\
MF13 & 390 & 4.8 & 4th WTGtr in WF1 A1 \\
MF14 & 390 & 4.7 & 7th, 8th and 9th WTGtr WF1 A2 \\
MF15 & 380 & 10.2 & 2nd and 3rd WTGtr in WF2 A1 \\
MF16 & 391 & 4.8 & 7th and 8th WTGtrs in WF1 A2 \\
MF17 & 385 & 4.5 & 1st, 3rd, and 5th WTGtrs in WF1 A1 \\
MF18 & 385 & 4.5 & 10th WTGtr in WF1 A1 \\
\hline \hline
\end{tabular}

trix D. Eigenvalues $\left\{\lambda_{i}=\sigma_{i} \pm j \omega_{i}\right\}_{1}^{n}$ and eigenvectors, $\phi_{i}$ : right eigenvector and $\psi_{i}$ : left eigenvector, are obtained using the command eig [34]. The frequency and \% damping ratio of a mode are found using the relations, $f=\omega / 2 \pi$ and $\zeta=-100 \sigma / \sqrt{\sigma^{2}+\omega^{2}}$, respectively. The relative participation of $k$ th state variable in $i$ th mode $p f_{\mathrm{ki}}$ is given as [35], $p f_{\mathrm{ki}}=\left(\left|\phi_{\mathrm{ik}}\right|\left|\psi_{\mathrm{ki}}\right|\right) /\left(\sum_{k=1}^{k=n}\left|\phi_{\mathrm{ik}}\right|\left|\psi_{\mathrm{ki}}\right|\right)$. In the presentation of results in the following section, vector of PF, $p f_{i}$, is normalized using the largest element in the vector.

\section{Modal Analysis of the AgGRegated System}

The AWS has 498 eigenvalues of which there are 173 complex pairs of eigenvalues with imaginary parts greater than $0.2 \mathrm{rad} / \mathrm{sec}$. There are 80 pairs of eigenvalues in the very high frequency range $(>3 \mathrm{kHz})$ and 14 pairs in the range of high frequency $(500 \mathrm{~Hz}$ to $3 \mathrm{KHz})$. The very high frequency and high frequency modes are not considered to be of importance in the current analysis because they are well damped and are not related to the dynamics under consideration. There are 18 modes in the frequency range $50 \mathrm{~Hz}$ to $500 \mathrm{~Hz}$ that are classified as MFMs as opposed to the three modes in FWS. There are also 16 stator modes having frequencies close to $50 \mathrm{~Hz}$. There are 45 modes in the low frequency range $(<50 \mathrm{~Hz})$ and they have very high damping ratio. The main focus of the paper is on the MFMs and the stator modes.

\section{A. Medium Frequency Modes}

Table II lists the frequency, damping ratio and the location of the participating states for the MFMs identified in the AWS model. Compared to the three MFMs observed in the FWS, the AWS contains 18 modes in the medium frequency range.

Table III shows the participating states of the MF1 and corresponding PF. The first column indicates the AWS element and state name. For example $W F T / i_{\mathrm{rD}}$ means inductor current in the WFT block. The second column refers to index (ID) of the element. For example, State $=W F T / i_{\mathrm{rD}}$, and ID $=2$, means 


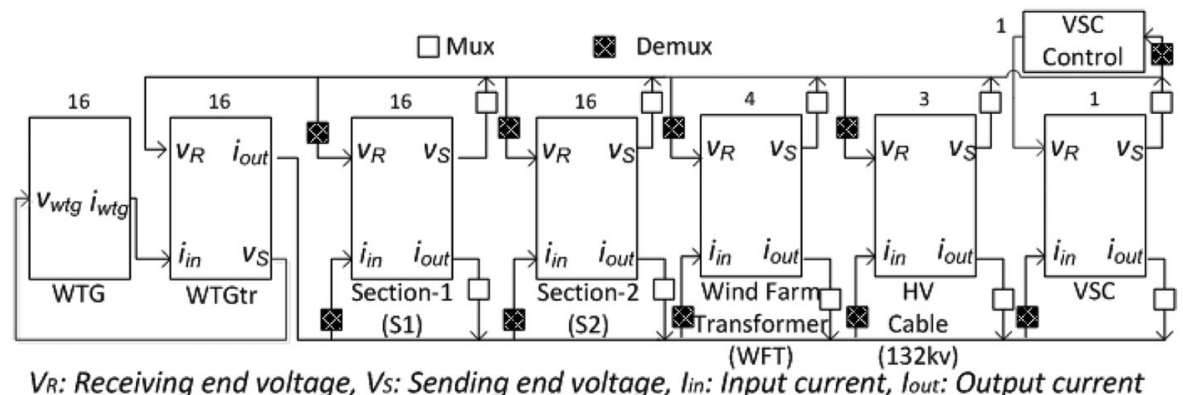

Fig. 5. Structure of wind farm simulation program.

TABLE III

CHARACTERISTICS OF THE MFM1

\begin{tabular}{|c|c|c|c|}
\hline State & ID & $\mathrm{PF}$ & Description \\
\hline$V S C / i_{\mathrm{rQ}}$ & 1 & 0.28 & $i_{\mathrm{rQ}}$ through VSC \\
\hline$W F T / i_{\mathrm{rQ}}$ & 1 & 0.31 & $i_{\mathrm{rQ}}$ through WF1 A1 WFT \\
\hline$W F T / i_{\mathrm{rQ}}$ & 2 & 0.31 & $i_{\mathrm{rQ}}$ through WF1 A2 WFT \\
\hline$W F T / i_{\mathrm{rD}}$ & 3 & 0.25 & $i_{\mathrm{rQ}}$ through WF2 A1 WFT \\
\hline$W F T / i_{\mathrm{rD}}$ & 4 & 0.25 & $i_{\mathrm{rD}}$ through WF2 A2 WFT \\
\hline$V S C / i_{\mathrm{rD}}$ & 1 & 0.37 & $i_{\mathrm{rD}}$ through VSC \\
\hline$V S C / v_{\mathrm{sQ}}$ & 1 & 0.71 & $v_{\mathrm{SQ}}$ at $\mathrm{PCC}$ \\
\hline$V S C / v_{\mathrm{sD}}$ & 1 & 1.00 & $v_{\mathrm{SD}}$ at $\mathrm{PCC}$ \\
\hline$W F T / i_{\mathrm{rD}}$ & 1 & 0.46 & $i_{\mathrm{rD}}$ through WF1 A1 WFT \\
\hline$W F T / i_{\mathrm{rD}}$ & 2 & 0.47 & $i_{\mathrm{rD}}$ through WF1 A2 WFT \\
\hline $132 \mathrm{kv} / v_{\mathrm{sQ}}$ & 1 & 0.20 & $v_{\mathrm{SQ}}$ at WF1 A1 WFT HV bus \\
\hline $132 \mathrm{kv} / v_{\mathrm{sQ}}$ & 2 & 0.20 & $v_{\mathrm{SQ}}$ at WF1 A2 WFT HV bus \\
\hline $132 \mathrm{kv} / v_{\mathrm{sD}}$ & 1 & 0.28 & $v_{\mathrm{SD}}$ at WF1 A1 WFT HV bus \\
\hline $132 \mathrm{kv} / v_{\mathrm{sD}}$ & 2 & 0.28 & $v_{\mathrm{sD}}$ at WF1 A2 WFT HV bus \\
\hline $132 \mathrm{kv} / v_{\mathrm{sD}}$ & 3 & 0.25 & $v_{\mathrm{sD}}$ at WF2 WFT HV bus \\
\hline
\end{tabular}

that the state is located at the second WFT which is in the WF1 $\mathrm{A} 2$. If $\mathrm{ID}=4$, it indicates to the state is located in the WFT in the WF2 A2. The states participating in the MF1 are located between the VSC and the WFTs.

The MF1 in the FWS [1] has frequency $=344.3 \mathrm{~Hz}$ (close to 7 th harmonics) and damping ratio $=7.47 \%$ compared to frequency $=369.3 \mathrm{~Hz}$ and damping ratio $=0.53 \%$ in the AWS. The frequency in both the systems are close though there is a big difference in the damping ratio. However, the participating states and their corresponding PFs are similar in both the cases. Since the aggregation method used in this work did not change the high voltage side of the wind farm, where the MF1 participating states are located, the state participation of the MF1 did not change. However, the aggregation of collector strings affected the damping ratio of the MF1.

Tables IV and $\mathrm{V}$ show the characteristics of the MF2, and the MF3, respectively, present in the AWS. Their frequencies are close to the 5 th, and 2 nd harmonics of the power frequency. The MF1 and the MF2 have participation from the current and voltage states in the VSC, $132 \mathrm{kV}$ cable and the WFTs. The MF3 has participation from states closer to the VSC. Modes with similar characteristics are observed in the FWS as well [1]. The MF2 has frequency $=243.4 \mathrm{~Hz}$ and damping ratio $=3.15 \%$ in the FWS compared to frequency $=257 \mathrm{~Hz}$ and damping ratio $=-3.2 \%$ in the AWS. Similarly, the MF3 has frequency $=99.7 \mathrm{~Hz}$ and damping ratio $=6.7 \%$ in the FWS compared to frequency $=117 \mathrm{~Hz}$ and damping ratio $=-19.8 \%$
TABLE IV

CHARACTERISTICS OF THE MFM2

\begin{tabular}{|c|c|c|c|}
\hline State & ID & $\mathrm{PF}$ & Description \\
\hline$V S C / i_{\mathrm{rQ}}$ & 1 & 0.60 & $i_{\mathrm{rQ}}$ through VSC \\
\hline$W F T / i_{\mathrm{rQ}}$ & 1 & 0.31 & $i_{\mathrm{rQ}}$ through WF1 A1 WFT \\
\hline$W F T / i_{\mathrm{rQ}}$ & 2 & 0.32 & $i_{\mathrm{rQ}}$ through WF1 A2 WFT \\
\hline$W F T / i_{\mathrm{rQ}}$ & 3 & 0.14 & $i_{\mathrm{rQ}}$ through WF2 A1 WFT \\
\hline$W F T / i_{\mathrm{rD}}$ & 3 & 0.18 & $i_{\mathrm{rD}}$ through WF2 A1 WFT \\
\hline$V S C / i_{\mathrm{rD}}$ & 1 & 0.32 & $i_{\mathrm{rD}}$ through VSC \\
\hline$V S C / v_{\mathrm{sQ}}$ & 1 & 1.00 & $v_{\mathrm{SQ}}$ at $\mathrm{PCC}$ \\
\hline$V S C / v_{\mathrm{sD}}$ & 1 & 0.60 & $v_{\mathrm{sD}}$ at $\mathrm{PCC}$ \\
\hline $\begin{array}{l}V S C \text { control } \\
\text { / stateD }\end{array}$ & 1 & 0.31 & VSC controller state \\
\hline $\begin{array}{l}V S C \text { control } \\
\text { / state } Q\end{array}$ & 1 & 0.18 & VSC controller state \\
\hline$W F T / i_{\mathrm{rD}}$ & 1 & 0.20 & $i_{\mathrm{rD}}$ through WF1 A1 WFT \\
\hline$W F T / i_{\mathrm{rD}}$ & 2 & 0.21 & $i_{\mathrm{rD}}$ through WF1 A2 WFT \\
\hline $132 k v / v_{\mathrm{sQ}}$ & 1 & 0.20 & $v_{\mathrm{SQ}}$ at WF1 A1 WFT HV bus \\
\hline $132 k v / v_{\mathrm{sQ}}$ & 2 & 0.21 & $v_{\mathrm{sQ}}$ at WF1 A2 WFT HV bus \\
\hline $132 k v / v_{\mathrm{sQ}}$ & 3 & 0.29 & $v_{\mathrm{sQ}}$ at WF 2 tr $\mathrm{HV}$ bus \\
\hline $132 k v / v_{\mathrm{sD}}$ & 1 & 0.18 & $v_{\mathrm{sD}}$ at WF1 A1 WFT HV bus \\
\hline $132 k v / v_{\mathrm{sD}}$ & 2 & 0.18 & $v_{\mathrm{sD}}$ at WF1 A2 WFT HV bus \\
\hline $132 k v / v_{\mathrm{sD}}$ & 3 & 0.17 & $v_{\mathrm{sD}}$ at $\mathrm{WF} 2 \operatorname{tr} \mathrm{HV}$ bus \\
\hline
\end{tabular}

TABLE V

CHARACTERISTICS OF THE MFM3

\begin{tabular}{llll}
\hline \hline State & ID & PF & Description \\
\hline$V S C / i_{\mathrm{rQ}}$ & 1 & 0.58 & $i_{\mathrm{rQ}}$ through VSC \\
$V S C / i_{\mathrm{rD}}$ & 1 & 1.00 & $i_{\mathrm{rD}}$ through VSC \\
$V S C / v_{\mathrm{sQ}}$ & 1 & 0.11 & $v_{\mathrm{sQ}}$ at PCC \\
$V S C / v_{\mathrm{sD}}$ & 1 & 0.19 & $v_{\mathrm{sD}}$ at PCC \\
$V S C$ control & 1 & 0.42 & VSC controller state \\
$/$ stateD & & & \\
VSCcontrol & 1 & 0.61 & \\
/stateQ & & & \\
\hline \hline
\end{tabular}

in the AWS. Interestingly, though the frequencies are close, the damping ratios the MF2 and the MF3 differ significantly between the AWS and the FWS.

The remaining 15 modes (MF4 to MF18) are not observed in FWS and they are grouped under the term synthetic modes in AWS. The analysis of synthetic modes are presented in Section III-B.

1) Effect of Operating Condition: The variation in the MFM frequency and damping for the following four test cases are analyzed. The results are compared with the corresponding results obtained from the FWS in [1]. 


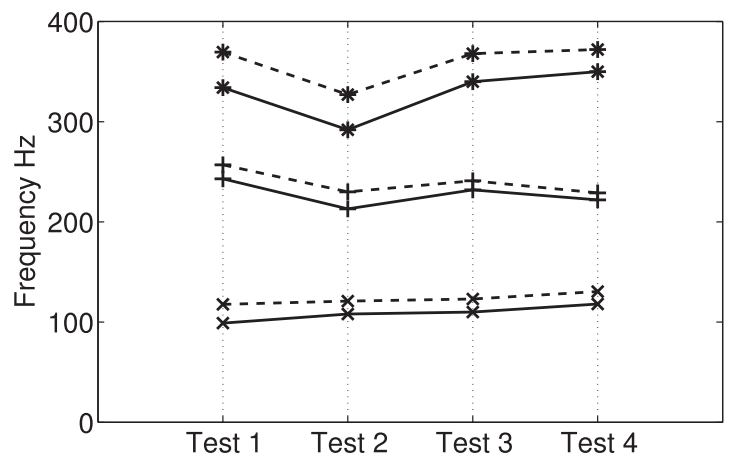

Fig. 6. Comparison of frequencies of the MFMs observed in the FWS and the AWS for different test conditions. Modes are marked using, MF1 : *, MF2 : +, MF3 : x. Straight lines connect the modes of the FWS and the dashed lines connect the modes of the AWS.

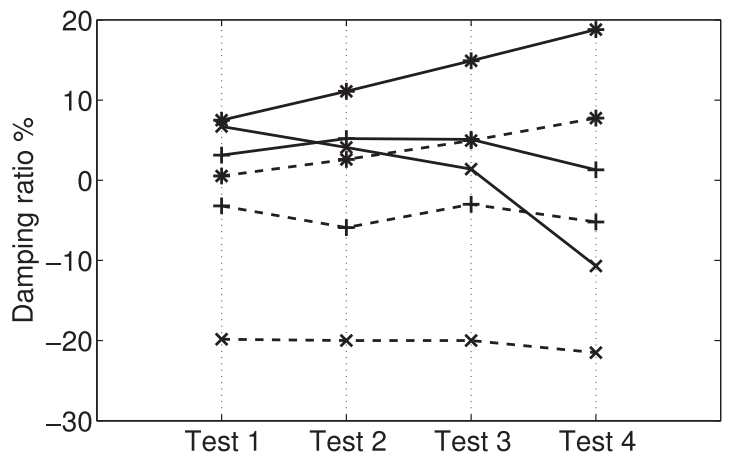

Fig. 7. Comparison of damping ratios of the MFMs observed in the FWS and the AWS for different test conditions. Modes are marked using, MF1 : *, MF2 : +, MF3 : x. Straight lines connect the modes of the FWS and the dashed lines connect the modes of the AWS.

Test-1: The base case where all the WTGs of both the wind farms are in service.

Test-2: The WF2 is partially shut down. Only five WTGs in the WF2 are working, which are located at the end of the strings. They are selected such that the entire $33-\mathrm{kV}$ collector cables remain energized.

Test-3: The WF1 A2 is partially shut down. Only eleven WTGs in the WF1 A2 are working which are located at the end of the strings. All the WTGs in the WF1 A1, and the WF2 are producing rated output.

Test-4: The WF1 A1 and the WF1 A2 are partially shut down. Only 10 WTGs in the WF1 A1 and 11 WTGs in the WF1 A2 are working which are located at the end of the strings. The WTGs in the WF2 are producing rated output.

Figs. 6 and 7 show the comparison of frequency and damping ratio for the MFMs observed in the FWS and the AWS. As seen from the figures, the frequency of the modes are quite close in both the system models. However, differences are observed in the damping ratios of the modes between the two system models.

2) Effect of VSC Tuning: The VSC controller transfer function in the FWS [1] is tuned such that the transfer function between the reference voltage and the PCC voltage has a gain cross over frequency of $100 \mathrm{~Hz}$. The controller tuning frequency is varied from $75 \mathrm{~Hz}$ to $175 \mathrm{~Hz}$ and the changes in the MFMs

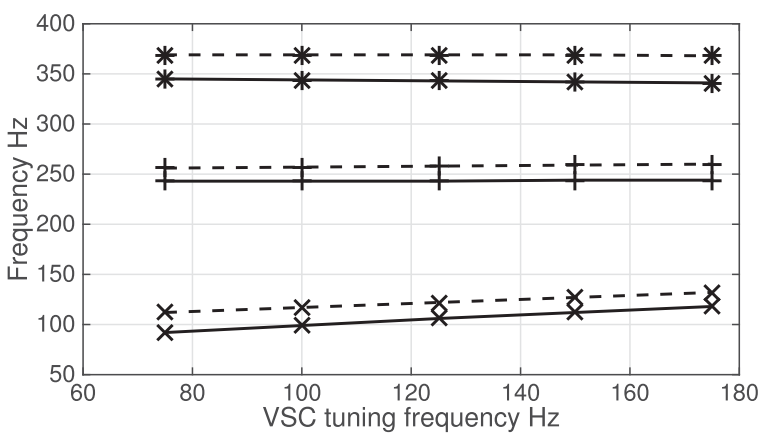

Fig. 8. Comparison of frequencies of the MFMs observed in the FWS and the AWS for different VSC tuning frequencies. Modes are marked using, MF1 : *, MF2 : +, MF3 : x. Straight lines connect the modes of the FWS and the dashed lines connect the modes of the AWS

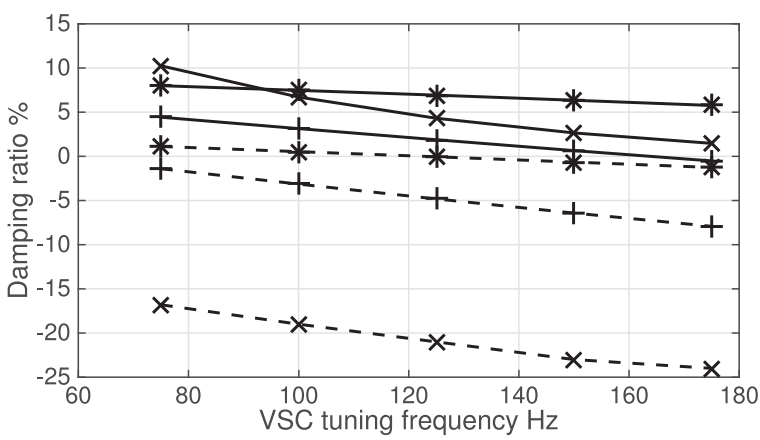

Fig. 9. Comparison of damping ratios of the MFMs observed in the FWS and the AWS for different VSC tuning frequencies. Modes are marked using, MF1 : *, MF2 : +, MF3 : x. Straight lines connect the modes of the FWS and the dashed lines connect the modes of the AWS

are observed. Figs. 8 and 9, respectively, show the variation in frequency and damping ratio for the MFMs with changes in the VSC controller tuning frequency for both the FWS and the AWS. The frequency and damping ratios of all three modes follow the same pattern of change as the controller tuning frequency is increased; however, a notable difference in the damping ratio exists for a similar controller tuning applied to the two systems. It is clear that the aggregated representation of the collector string reduces the damping of the MFMs.

\section{B. Characteristics of the Synthetic Modes}

There are 15 additional modes in the medium frequency range that have been observed only in the AWS. Their frequency and damping ratio are close to the MFMs observed in the FWS. The characteristics of two of the synthetic modes, MF6 and MF12, are shown in Tables VI and VII, respectively. The MF6 has participation from the states of all WTGtrs of the WF1 except WTG6 and WTG10 (both machines are comparatively small), the WFTs in the WF1 and the $132 \mathrm{kV}$ cables connecting the WF1 and the PCC. The MF12 has participation from the states, the WTG terminal voltage and the WTGtr currents of the WTG9 and the WTG11. Other synthetic modes also exhibit similar participation from the states located at the $33 \mathrm{kV}$ side of the AWS. These modes are the result of aggregating cable parameters and representing them as lumped elements. One way to support this interpretation is to consider the inertia 
TABLE VI

CHARACTERISTICS OF THE MF6

\begin{tabular}{lccc}
\hline \hline State & ID & PF & Description \\
\hline$W F T / i_{\mathrm{rQ}}$ & 1 & 0.62 & $i_{\mathrm{rQ}}$ through WF1 A1 WTF \\
$W F T / i_{\mathrm{rQ}}$ & 2 & 0.58 & $i_{\mathrm{rQ}}$ through WF1 A2 WFT \\
$132 k v / i_{\mathrm{rD}}$ & 1 & 0.25 & $i_{\mathrm{rD}}$ through WF1 A1 132kv \\
$132 k v / i_{\mathrm{rD}}$ & 2 & 0.24 & $i_{\mathrm{rD}}$ through WF1 A2 132kv \\
$W F T / i_{\mathrm{rD}}$ & 1 & 1.00 & $i_{\mathrm{rD}}$ through WF1 A1 WFT \\
$W F T / i_{\mathrm{rD}}$ & 2 & 0.92 & $i_{\mathrm{rD}}$ through WF1 A2 WFT \\
$W T G t r / v_{\mathrm{sd}}$ & 1 & 0.32 & $v_{\mathrm{sD}}$ at WTG-1 terminal \\
$W T G t r / v_{\mathrm{sd}}$ & 2 & 0.29 & $v_{\mathrm{sD}}$ at WTG-2 terminal \\
$W T G t r / v_{\mathrm{sd}}$ & 3 & 0.32 & $v_{\mathrm{sD}}$ at WTG-3 terminal \\
$W T G t r / v_{\mathrm{sd}}$ & 4 & 0.24 & $v_{\mathrm{sD}}$ at WTG-4 terminal \\
$W T G t r / v_{\mathrm{sd}}$ & 5 & 0.32 & $v_{\mathrm{sD}}$ at WTG-5 terminal \\
$W T G t r / v_{\mathrm{sd}}$ & 7 & 0.24 & $v_{\mathrm{sD}}$ at WTG-7 terminal \\
$W T G t r / v_{\mathrm{sd}}$ & 8 & 0.24 & $v_{\mathrm{sD}}$ at WTG-8 terminal \\
$W T G t r / v_{\mathrm{sd}}$ & 9 & 0.28 & $v_{\mathrm{sD}}$ at WTG-9 terminal \\
$W T G t r / v_{\mathrm{sd}}$ & 11 & 0.31 & $v_{\mathrm{sD}}$ at WTG-11 terminal \\
\hline \hline
\end{tabular}

TABLE VII

CHARACTERISTICS OF THE MF12

\begin{tabular}{lccc}
\hline \hline State & ID & PF & Description \\
\hline$W T G t r / i_{\mathrm{rQ}}$ & 11 & 0.55 & $i_{\mathrm{rQ}}$ through WTGtr-11 \\
$W T G t r / i_{\mathrm{rD}}$ & 9 & 0.25 & $i_{\mathrm{rD}}$ through WTGtr-9 \\
$W T G t r / i_{\mathrm{rD}}$ & 11 & 0.92 & $i_{\mathrm{rD}}$ through WTGtr-11 \\
$W T G t r / v_{\mathrm{sq}}$ & 11 & 0.59 & $v_{\mathrm{sQ}}$ at WTG-11 terminal \\
$W T G t r / v_{\mathrm{sd}}$ & 9 & 0.26 & $v_{\mathrm{sD}}$ at WTG-9 terminal \\
$W T G t r / v_{\mathrm{sd}}$ & 11 & 1.00 & $v_{\mathrm{sD}}$ at WTG-11 terminal \\
\hline \hline
\end{tabular}

TABLE VIII

CHARACTERISTICS OF THE STATOR MODES IN THE AWS

\begin{tabular}{lccc}
\hline \hline State & ID & PF & Description \\
\hline$W T G / e_{\mathrm{qs}}$ & 3 & 0.60 & WTG-1 stator $i_{\mathrm{qs}}$ \\
$W T G / e_{\mathrm{qs}}$ & 5 & 1.00 & WTG-3 stator $i_{\mathrm{qs}}$ \\
$W T G / i_{\mathrm{ds}}$ & 3 & 0.53 & WTG-1 stator $i_{\mathrm{ds}}$ \\
$W T G / i_{\mathrm{ds}}$ & 5 & 0.89 & WTG-3 stator $i_{\mathrm{ds}}$ \\
$W T G / e_{\mathrm{qs}}$ & 3 & 0.58 & WTG-4 stator $i_{\mathrm{ds}}$ \\
$W T G / e_{\mathrm{qs}}$ & 5 & 0.96 & WTG-1 stator $e_{\mathrm{ds}}$ \\
\hline
\end{tabular}

(H constant) of the equivalent DFIG to represent a string. Typically ten single DFIG when aggregated has $50 \mathrm{MW}$ capacity and ten times higher inertia. This moves some of the high frequency modes in the FWS towards the range of the MFMs. Because of this, additional 15 modes are appeared in the AWS which are merely the result of aggregation and will not exist in reality. The conclusions arrived based on the AWS should make clear that these modes are not present in the FWS and make their influence irrelevant.

\section{Characteristics of Stator Modes}

The AWS contains 16 stator modes corresponding to 16 WTGs. They have poor damping ratios and frequencies close to the power system frequency. Characteristics of one of the stator modes is shown in Table VIII where the mode has participation from the states of three of the WTGs. Similar to the FWS, the stator modes also have participation from one or more WTGs. In the FWS, the stator modes have frequencies close to $49.25 \mathrm{~Hz}$ $( \pm 0.05)$ and a damping ratio $1.65 \%( \pm 0.02)$ whereas, in the

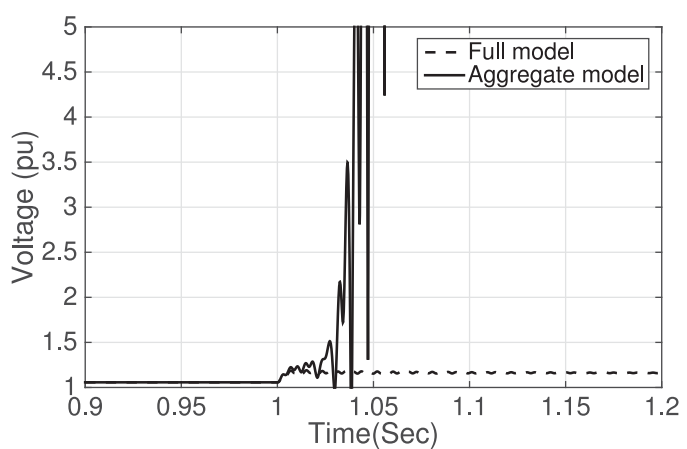

Fig. 10. Plot of the PCC voltage following a change in the reactive power reference of the WTGs in the AWS.

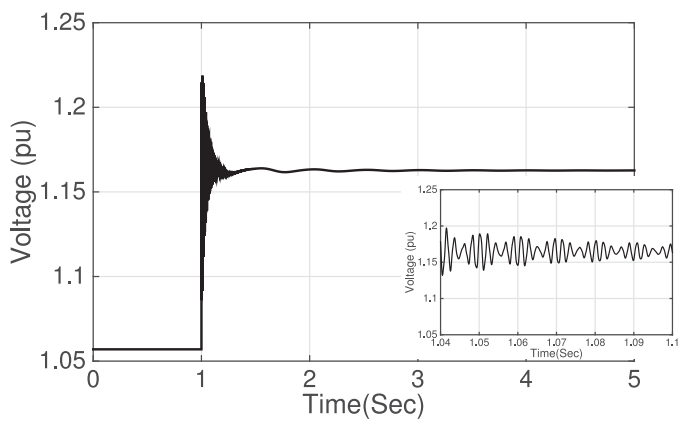

Fig. 11. PCC voltage following a $10 \%$ increase in the PCC reference voltage using the FWS. Inset shows magnified view of the plot.

AWS, they are $48.89 \mathrm{~Hz}( \pm 0.1)$ and have a damping ratio of $0.96 \%( \pm 0.03)$, respectively.

\section{Dynamic Simulation of Aggregated Wind FARM MODEL}

The modal analysis results on the AWS presented so far have shown that aggregation has changed the damping ratios of the MFM and introduced many synthetic modes. Fig. 10 shows the PCC voltage following a change in the reactive power reference input of the WTGs in the FWS and the AWS. Due to the negative damping of the MFMs, simulation of the AWS produces an unstable response. The simulation failed to continue to the end of the study period.

In order to gain an understanding of the performance of the aggregated system, it is desirable to get response where simulation can continue till the end of the simulation duration. The VSC controller is retuned to improve the damping of MFM in both the FWS and the AWS. A step change in the VSC reference input is applied to both the systems with the new controller and the results are presented in the next subsection for comparison.

\section{A. $10 \%$ Increase in VSC Reference Voltage}

Figs. 11-16 show various voltages in the systems following a step change in VSC reference input at time $t=1 \mathrm{sec}$.

Oscillations in the MFM range are visible in the PCC voltage obtained from both the systems (Figs. 11 and 12). As expected, the settling time of the oscillations in the AWS is more due to poor damping of the MFM. The voltage at the $33 \mathrm{kV}$ bus of the 


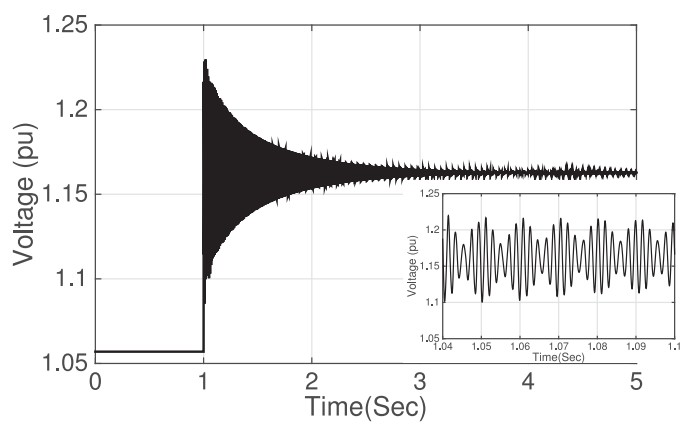

Fig. 12. PCC voltage following a $10 \%$ increase in the PCC reference voltage using the AWS. Inset shows magnified view of the plot.

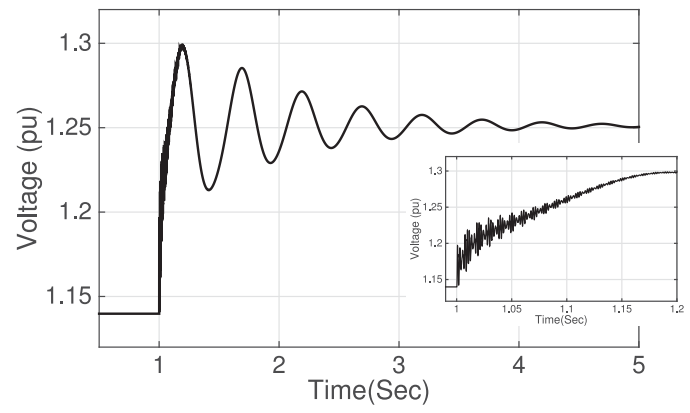

Fig. 13. Voltage at the WF1 A1 WFT $33 \mathrm{kV}$ bus following a $10 \%$ increase in the PCC reference voltage using the FWS. Inset shows magnified view of the plot.

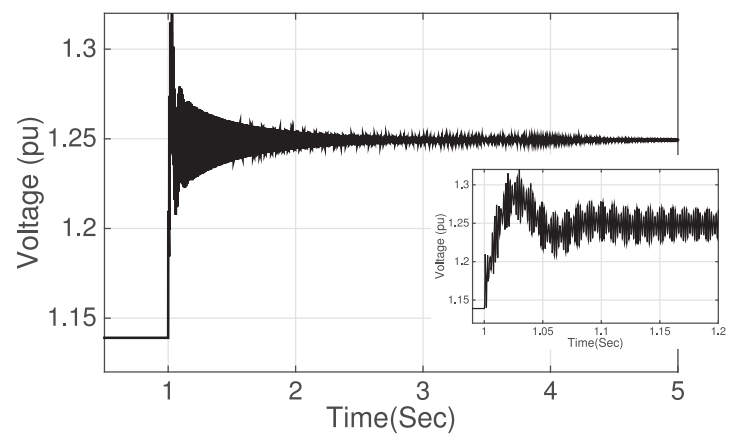

Fig. 14. Voltage at the WF1 A1 WFT $33 \mathrm{kV}$ bus following a $10 \%$ increase in the PCC reference voltage using the AWS. Inset shows magnified view of the plot.

WF1 A1 WFT in the FWS and the AWS are shown in Figs. 13 and 14, respectively. The MFM oscillations are not visible in the voltage waveform of the FWS. However, in the AWS, the waveform is distorted due to the presences of the MFMs and the synthetic modes.

Similarly, voltage at the terminal of the WTG1 obtained from the FWS and the AWS are shown in Figs. 15 and 16, respectively. The waveform is free from any MFM oscillations in the FWS compared to the severely distorted waveform in the AWS. The WTG terminal voltage in the AWS settles faster than the FWS because of the aggregated control action of the aggregated WTG than several WTGs forming a string in the FWS. It is to be noted that in the AWS the low frequency oscillations at the WTG terminal voltage appears to settle faster, but in reality the oscillations prolong for around $3 \mathrm{sec}$ as observed in the

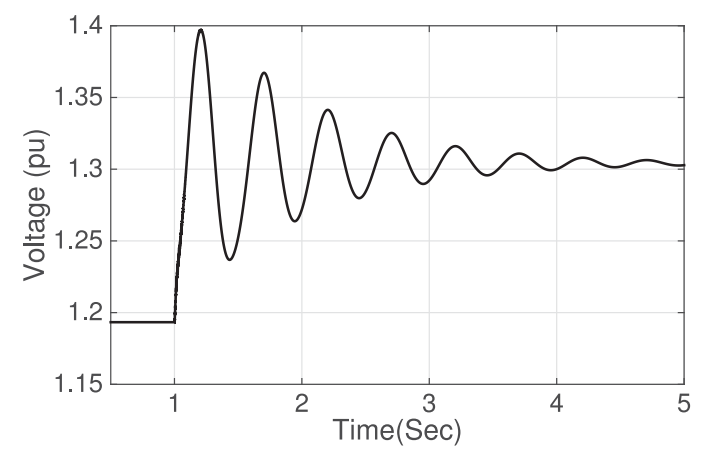

Fig. 15. Voltage at the WTG1 terminal following a $10 \%$ increase in the PCC reference voltage using the FWS.

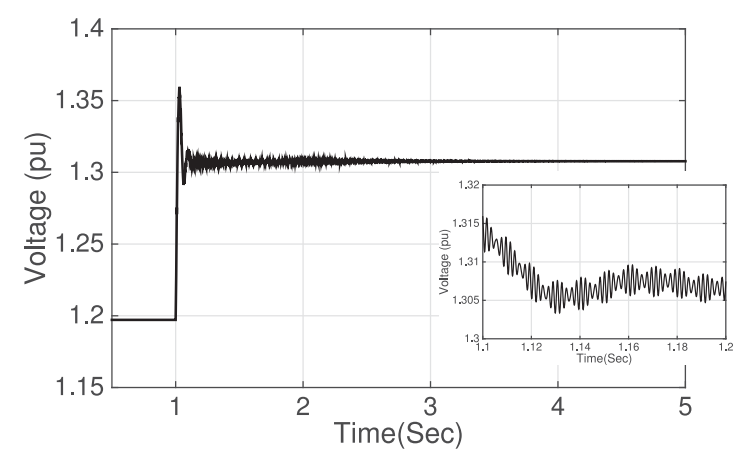

Fig. 16. Voltage at the WTG1 terminal following a 10\% increase in the PCC reference voltage using the AWS. Inset shows magnified view of the plot.

FWS. Similarly, oscillations in the range of power frequency harmonics are visible at the WTG terminal in the AWS simulation results which is a concern for converter designers, but is not present in the simulation results obtained from the FWS. It is clear that the aggregation of the WTGs alters characteristics of the complete system and due care must be taken while interpreting the results.

\section{CONCLUSION}

In this paper, the order of the wind farm system is reduced by aggregating some of the WTGs and the collector system cables in order to analyze the consequences of aggregation on the critical modes of system resonance. The dynamic aggregation of the system does simplify the computational complexity and the frequency characteristics of the critical modes are preserved in the aggregation, but it does produce significantly different values of damping to the modes close to the $2 \mathrm{nd}$, 5th and 7 th harmonics of the power system frequency. Both frequency and time domain analysis confirm this. Also, more MFMs in the region of $50 \mathrm{~Hz}$ to $500 \mathrm{~Hz}$, the "synthetic modes," appear because of the equivalencing. In some operating conditions damping ratios are even negative giving a qualitatively different assessment of stability conditions when compared with that obtained from a detailed and complex model reported in [1]. The dynamic simulation results obtained from the FWS and the AWS show differences in voltage waveforms at different buses of the system. This clearly suggests that the existing practice of dynamic equivalencing is not adequate. It is required to explore 
improved aggregation methods. Our immediate future research is undertaking this task.

\section{REFERENCES}

[1] L. P. Kunjumuhammed, B. C. Pal, C. Oates, and K. Dyke, "Electrical oscillations in wind farm systems: Analysis and insight based on detailed modelling," IEEE Trans. Sustain. Energy, vol. 7, no. 1, pp. 51-62, Jan. 2016.

[2] E. Muljadi et al., "Equivalencing the collector system of a large wind power plant," in Proc. IEEE Power Eng. Soc. General Meeting, 2006, pp. 1-9.

[3] G. Quinonez-Varela, G. Ault, O. Anaya-Lara, and J. McDonald, "Electrical collector system options for large offshore wind farms," IET Renewable Power Gener., vol. 1, no. 2, pp. 107-114, Jun. 2007.

[4] W. Lin, J. Wen, J. Liang, S. Cheng, M. Yao, and N. Li, "A three-terminal HVDC system to bundle wind farms with conventional power plants," IEEE Trans. Power Syst., vol. 28, no. 3, pp. 2292-2300, Aug. 2013.

[5] S. Dutta and T. Overbye, "A clustering based wind farm collector system cable layout design," in Proc. IEEE Power Energy Conf., IL, USA, Feb. 2011, pp. 1-6.

[6] S. Kuenzel, L. P. Kunjumuhammed, B. C. Pal, and I. Erlich, "Impact of wakes on wind farm inertial response," IEEE Trans. Sustain. Energy, vol. 5, no. 1, pp. 237-245, Jan. 2014.

[7] A. Marinopoulos et al., "Investigating the impact of wake effect on wind farm aggregation," in Proc. IEEE PowerTech, Trondheim, Norway, Jun. 19-23, 2011, pp. 1-5.

[8] J. K. Sethi, D. Deb, and M. Malakar, "Modeling of a wind turbine farm in presence of wake interactions," in Proc. Int. Conf. Energy, Autom. Signal, Dec. 28-30, 2011, pp. 1-6.

[9] G. Tapia, A. Tapia, and J. X. Ostolaza, "Two alternative modeling approaches for the evaluation of wind farm active and reactive power performances," IEEE Trans. Energy Convers., vol. 21, no. 4, pp. 909-920, Dec. 2006.

[10] G. Tapia, A. Tapia, and J. X. Ostolaza, "Two alternative modeling approaches for the evaluation of wind farm active and reactive power performances," IEEE Trans. Energy Convers., vol. 21, no. 4, pp. 909-920, Dec. 2006.

[11] V. Akhmatov and H. Knudsen, "An aggregate model of a grid-connected, large-scale, offshore wind farm for power stability investigations: Importance of windmill mechanical system," Int. J. Elect. Power Energy Syst., vol. 24, no. 9, pp. 709-717, Nov. 2002.

[12] A. Perdana and C. Ola, "Aggregated models of a large wind farm consisting of variable speed wind turbines for power system stability studies," in Proc. 8th Int. Workshop Large-Scale Integr. Wind Power Power Syst. Well Transmiss. Netw. Offshore Wind Farms, pp. 568-573, 2009.

[13] T. Xin, H. Shen, H. Bao, B. Huang, D. Chen, and J. Ding, "A practical equivalence method of large scale wind farm," in Proc. Int. Conf. Power Syst. Technol, Oct. 24-28, 2010, pp. 1-6.

[14] H. Liu and Z. Chen, "Aggregated modelling for wind farms for power system transient stability studies," in Proc. Asia-Pacific Power Energy Eng. Conf., Mar. 2012, pp. 1-6.

[15] L. M. Fernandez, C. A. Garcia, F. Jurado, and J. R. Saenz, "Aggregation of doubly fed induction generators wind turbines under different incoming wind speeds," in Proc. IEEE Russia Power Tech, Jun. 27-30, 2005, pp. $1-6$.

[16] M. A. Chowdhury, N. Hosseinzadeh, M. M. Billah, and S. A. Haque, "Dynamic DFIG wind farm model with an aggregation technique" in Proc. Int. Conf. Elect. Comput. Eng., Dec. 18-20, 2010, pp. 330-333.

[17] N. Dhlamini and S. P. Chowdhury, "The impact of wind farm aggregation techniques for analyzing power system dynamics," in Proc. 50th Int. Univ. Power Eng. Conf., Sep. 1-4, 2015 pp. 1-6.

[18] L. M. Fernndez, J. Francisco, and J. R. Saenz, "Aggregated dynamic model for wind farms with doubly fed induction generator wind turbines," Renewable Energy, vol. 33, no. 1, pp. 129-140 Jan. 2008.

[19] M. A. Chowdhury, W. X. Shen, N. Hosseinzadeh, and H. R. Pota, "A novel aggregated DFIG wind farm model using mechanical torque compensating factor," Energy Convers. Manage., vol. 67, pp. 265-274, Mar. 2013.

[20] M. Abbes, M. Allagui, and O. B. K. Hasnaoui, "An aggregate model of PMSG-based, grid connected wind farm: Investigation of LVRT capabilities," in Proc. 6th Int. Renewable Energy Congr., Mar. 24-26, 2015, pp. 1-6.

[21] H. Q. Zhou, Z. P. Song, J. P. Wang, and Y. Xue, "A review on dynamic equivalent methods for large scale wind farms," in Proc. Asia-Pacific Power Energy Eng. Conf., Mar. 25-28, 2011, pp. 1-7.
[22] Y. Cheng, M. Sahni, J. Conto, S. H. Huang, and J. Schmall, "Voltageprofile-based approach for developing collection system aggregated models for wind generation resources for grid voltage ride-through studies," IET Renewable Power Gener, vol. 5, no. 5, pp. 332-346, Sep. 2011.

[23] L. Li, D. Kui, T. Juan, W. Ningbo, and M. Yanhong, "Coherency-based dynamic equivalence method for power system centralized large scale wind power," in Proc. IEEE Int. Conf. Power Syst. Technol., Oct. 30 2012-Nov. 2, 2012, pp. 1-6.

[24] K. Rudion, Z. Styczynski, A. Orths, and O. Ruhle, "MaWind-Tool for the aggregation of wind farm models," in Proc. IEEE Power Energy Soc. General Meeting - Convers. Del. Elect. Energy 21st Century, Jul. 20-24, 2008, pp. 1-8.

[25] K. Dong-Eok and M. A. El-Sharkawi, "Dynamic equivalent model of wind power plant using an aggregation technique," IEEE Trans. Energy Convers., vol. 30, no. 4, pp. 1639-1649, Dec. 2015.

[26] M. Ali, I.-S. Ilie, J. Milanovic, and G. Chicco, "Wind farm model aggregation using probabilistic clustering," IEEE Trans. Power Syst., vol. 28 , no. 1, pp. 309-316, Feb. 2013.

[27] L. M. Fernndez, C. A. Garca, J. R. Saenz, and F. Jurado, "Equivalent models of wind farms by using aggregated wind turbines and equivalen winds," Energy Convers. Manage., vol. 50, no. 3, pp. 691-704, Mar. 2009.

[28] L. M. Fernandez, C. A. Garcia, J. R. Saenz, and F. Jurado, "Reduced model of DFIGs wind farms using aggregation of wind turbines and equivalent wind," in Proc. IEEE Mediterranean Electrotech. Conf, May. 16-19, 2006 pp. 881-884.

[29] M. A. El-Sharkawi, "Dynamic equivalent models for wind power plants," in Proc. IEEE Power Energy Soc. General Meeting, Jul. 24-29, 2011, pp. $1-5$.

[30] G. Sudipta and S. Nilanjan, "Balanced truncation based reduced order modeling of wind farm," Int. J. Elect. Power Energy Syst., vol. 53 pp. 649-655, Dec. 2013.

[31] H. Li, C. Yang, B. Zhao, H. S. Wang, and Z. Chen, "Aggregated models and transient performances of a mixed wind farm with different wind turbine generator systems," Electric Power Syst. Res., vol. 92, pp. 1-10, Nov. 2012

[32] I. Erlich, F. Shewarega, C. Feltes, F. Koch, and J. Fortmann, "Determination of dynamic wind farm equivalents using heuristic optimization," in Proc. IEEE Power Energy Soc. General Meeting , Jul. 22-26, 2012, pp. 1-8.

[33] L. Xu, L. Yao, and C. Sasse, "Grid integration of large DFIG-based wind farms using VSC transmission," IEEE Trans. Power Syst., vol. 22, no. 3 pp. 976-984, Aug. 2007.

[34] Online Technical Documentation of MATLAB, The MathWorks Inc. Natick, MA, USA, 2015. [Online]. Available: http://www.mathworks .co.uk/help/techdoc/

[35] B. Pal and B. Chaudhuri, Robus Control in Power Systems. New York, NY, USA: Springer, 2005

Linash Puthenpurayil Kunjumuhammed (S'01-M'13-SM'16) received the B.Tech. degree from Mahatma Gandhi University, Kottayam, India, the M.S degree from the Indian Institute of Technology Madras, Chennai, India, and the Ph.D. degree from Imperial College London, London, U.K., in 2002, 2006, and 2012, respectively, all in electrical engineering.

He was a Graduate Apprentice Trainee with Neyveli Lignite Corporation Ltd., Neyveli, India, from September 2002 to August 2003. In 2006, he joined the Advanced Engineering Group, TVS Motor Company Ltd., Hosur, India, and in 2008, he joined Imperial College London as a Research Assistant. From September 2012 to February 2013, he was with GE Global Research-Europe, Munich, Germany, as a Marie Curie Research Fellow, Secondment from Imperial College London, under the REAL-SMART project funded by the Marie Curie FP7 IAPP scheme. Since 2013, he has been working as a Research Associate at Imperial College London. His research interests include power system dynamics stability and control, renewable electricity generation, and wind farm dynamics. 
Bikash Chandra Pal (M'00-SM'02-F'13) received 15 Ph.D. degrees. He is currently a Professor of power systems at Imperial College London, London, U.K. His research interest includes power system stability, control and computation. He has published 60 technical papers in IEEE transactions and IET journals. He has coauthored two books and two award winning IEEE Task Force/Working Group reports. He is Editor-in-Chief of IEEE TRANSACTIONS ON SUSTAINABLE ENERGY.

Colin Oates (M'05) was born in Yorkshire, U.K., in 1950. He received the B.Sc. degree in electrical and electronic engineering from Hertfordshire University, Hatfield, U.K., in 1973, and the M.Sc. and Ph.D. degrees in electrical engineering from Manchester University, Manchester, U.K., in 1975 and 1981, respectively.

He was an Apprentice and Junior Engineer with British Aerospace, Hatfield, U.K., between 1969 and 1974, and later joined ALSTOM Grid, Stafford, U.K., in 1981, as a Senior Development Engineer. In 1987, he became Group Head of the Power Electronics Group, ALSTOM Research and Technology Centre. In 2007, he transferred to ALSTOM Power Electronics Systems to develop VSC systems for power transmission. He has the expert status within ALSTOM of Senior Fellow and is an Honorary Associate Professor with Nottingham University, Nottingham, U.K. He has worked on projects such as the MAGLEV shuttle development for Birmingham Airport in the 1980s, and power converters for CERN, JET, and the U.K. AEA Culham Laboratory. He has worked on Traction projects, being involved in the development of interference current monitor equipment for ALSTOM Transport and has worked on projects involving ac and dc high-voltage transmission. Until recently, he was involved in the development of voltage source converters for HVDC applications. He has authored more than 30 papers on electronics and power electronics. Very sadly and suddenly he passed away on 25th January, 2016.
Kevin J. Dyke (M'05) received the Graduate degree from the University of Manchester Institute of Science and Technology, Manchester, U.K., in 2005, and the M.Eng degree in electrical and electronic engineering. Following this, he received a Postgraduate Diploma in management science from Manchester Business School, Manchester, U.K., in 2007 and an Engineering Doctorate, Eng.D, from the University of Manchester in 2009. He is currently a Chartered Engineer with the IET, ILM, and STEMnet. He was the HVDC Research and Technology Manager at GE Grid Solutions, Stafford, U.K. (previously known as ALSTOM Grid). He is currently the Manager with the HVDC Valves Department, Stafford, U.K. 